\title{
Application of high resolution T1 mapping with MOLLI (hrMOLLI) to differentiate patients with diffuse and regional myocardial disease from healthy subjects
}

\author{
Ana Pastor ${ }^{1}$, Zhong Chen ${ }^{1}$, Tobias Voigt ${ }^{2}$, Reza Razavi ${ }^{1}$, Tobias Schaeffter ${ }^{1}$, Eike Nagel ${ }^{1}$, Valentina O Puntmann ${ }^{1 *}$ \\ From 15th Annual SCMR Scientific Sessions \\ Orlando, FL, USA. 2-5 February 2012
}

\section{Summary}

Cardiac magnetic resonance (CMR) is increasingly applied as the first line investigation into the causes of myocardial dysfunction and remodelling. Although regional fibrosis is easily imaged on late gadolinium enhancement, characterisation of myocardial conditions with diffuse fibrosis remains challenging. Several studies proposed T1 mapping of gadolinium-enhanced myocardium supported by an inverse relationship between $\mathrm{T} 1$ relaxation times and amount of fibrotic tissue on histology. We performed high-resolution modified LookLocker inversion recovery (hrMOLLI) imaging of native and postcontast myocardium in thirty-six subjects who underwent a routine CMR protocol for investigation of cardiomyopathy.

\section{Background}

Several studies proposed T1 mapping of gadoliniumenhanced myocardium supported by an inverse relationship between $\mathrm{T} 1$ relaxation times and amount of fibrotic tissue on histology. To date no studies characterised the T1 values in non-enhanced myocardium in conditions with diffuse and regional fibrosis, nor evaluated its relation to post-contrast $\mathrm{T} 1$ values consistently in a clinical setting.

\section{Methods}

We performed high-resolution modified Look-Locker inversion recovery (hrMOLLI) imaging of native and postcontrast myocardium in thirty-six subjects (age

${ }^{1}$ Cardiovascular Imaging, King's College London, London, UK Full list of author information is available at the end of the article
$($ mean \pm SD) $47 \pm 7.4$ years) who underwent a routine CMR protocol for investigation of cardiomyopathy (hypertrophic cardiomyopathy: $\mathrm{n}=6$; longstanding hypertension: $n=7$, previous myocardial infarction: $n=12$ ). Eleven subjects referred for exclusion of arythmogenic right ventricular cardiomyopathy with normal CMR findings served as controls. Single breath-hold hrMOLLI was performed on a $3 \mathrm{~T}$ clinical scanner in an equatorial short axis slice prior and at 10, 20 and 40 minutes following administration of $0.2 \mathrm{mmol} / \mathrm{kg}$ gadoliniumDTPA contrast. Imaging parameters were FOV 320x320; TR/TE $3.2 / 1.57 \mathrm{~ms}$, flip angle $50^{\circ}$, interpolated voxel size $0.9 \times 0.9 \times 8 \mathrm{~mm}$, phase encoding steps $\mathrm{n}=166$, trigger delay: $450 \mathrm{msec}$. T1 map values were obtained by placing the region of interest (ROI) within the septal myocardium. In patients with myocardial scar on late gadolinium enhancement, ROIs were derived from both remote and scarred areas pre-contrast and 20 minutes afterwards only. In addition to the native and postcontrast $\mathrm{T} 1$ values the ratio between postcontrast and precontrast values was calculated.

\section{Results}

Native T1 relaxation times were shorter in healthy myocardium than in diffuse fibrosis or scarred areas (Figure $1)$, whereas $\mathrm{T} 1$ values in remote areas did not differ significantly from healthy myocardium. Postcontrast T1 values were significantly shorter in diffuse fibrosis at 5 20 minutes $(\mathrm{p}<0.05)$, but not at 40 minutes post injection. At 20 minutes, T1 values in scarred areas were significantly shorter than in healthy myocardium or diffuse fibrosis $(\mathrm{p}<0.01)$. The relative ratio between postcontrast and precontrast values showed significantly higher 


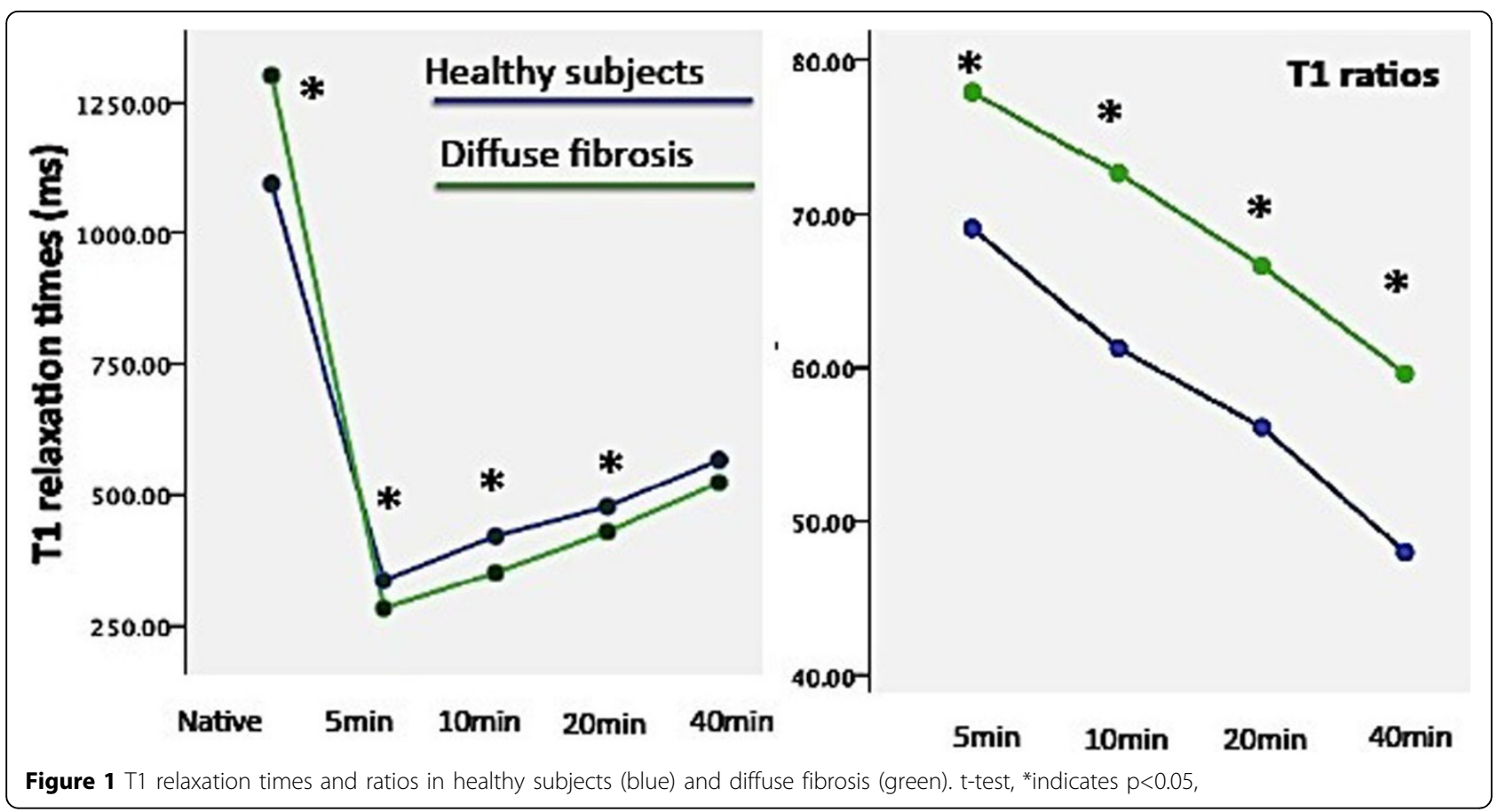

values for diffuse fibrosis in comparison to normal myocardium at all time-points (5-40min). For post contrast myocardium a cut-off value of $320 \mathrm{~ms} 10 \mathrm{~min}$ post injection provided the greatest distinction between healthy myocardium and diffuse fibrosis (sensitivity $100 \%$, specificity $83 \%$, AUC $=0.85,95 \% \mathrm{CI}: 0.68-1, \mathrm{p}=0.004$ ) (Figure 2). Distinction of normal and fibrotic tissue was

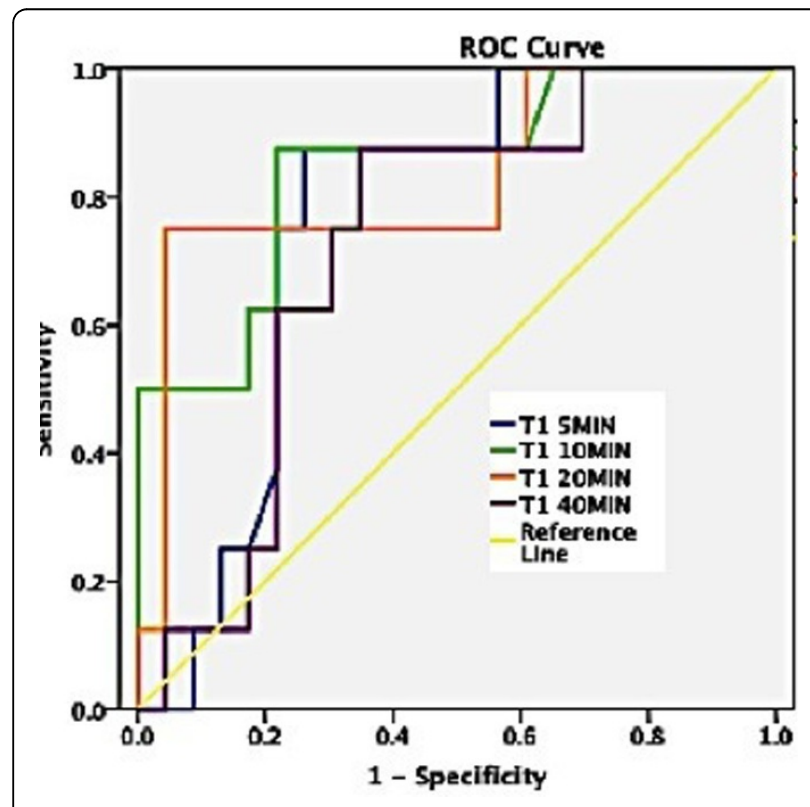

Figure 2 ROC analysis reveals a cut-off value of 320 ms 10min post injection provided the greatest distinction between healthy myocardium and diffuse fibrosis. significantly better with native than with contrast enhanced images $(\mathrm{p}<0.001)$.

\section{Conclusions}

We demonstrate that T1 mapping of native and postcontrast myocardium can serve to differentiate between healthy myocardium and diffuse fibrosis with better accuracy than after contrast injection.

\section{Funding}

NIHR British Research Centre.

\section{Author details}

${ }^{1}$ Cardiovascular Imaging, King's College London, London, UK. ²Philips

Research, London, UK.

Published: 1 February 2012

doi:10.1186/1532-429X-14-S1-P225

Cite this article as: Pastor et al:: Application of high resolution T1 mapping with MOLLI (hrMOLLI) to differentiate patients with diffuse and regional myocardial disease from healthy subjects. Journal of Cardiovascular Magnetic Resonance 2012 14(Suppl 1):P225. 